\title{
The role of free immunoglobulin light chains in serum from SLE patients with or without renal involvement
}

\author{
Adriana Aguirre Telio ${ }^{1 \S}$, Citlaly López Orozco ${ }^{2 \S}$, Susana Maricela Hernández Doño ${ }^{2}$, Daniela Ruiz Gómez ${ }^{3}$, \\ Víctor Hugo Tovar Méndez ${ }^{4}$, María Fernanda García Gutiérrez ${ }^{4}$, Miriam Santiago Ortíz ${ }^{5}$, Marcos Meneses \\ Mayo ${ }^{5}$, Antonio Villa-Romero ${ }^{6}$, Julio Granados ${ }^{2 *}$ \\ ${ }^{1}$ Central Laboratory, Molecular Biology Area, Instituto Nacional de Ciencias Medicas y Nutricion Salvador Zubiran, Mexico City 14000, Mexico. \\ ${ }^{2}$ Transplant Department, Instituto Nacional de Ciencias Medicas y Nutricion Salvador Zubiran, Mexico City 14000, Mexico. \\ ${ }^{3}$ Fundacion Clinica Medica Sur, Mexico City 14000, Mexico. \\ ${ }^{4}$ Instituto Nacional de Ciencias Medicas y Nutricion Salvador Zubiran, Mexico City 14000, Mexico. \\ ${ }^{5}$ Universidad Anahuac, Facultad de Ciencias de la Salud, Huixquilucan, State of Mexico 14000, Mexico. \\ ${ }^{6}$ Research Department. Universidad Panamericana, Mexico City 14000, Mexico.
}

\begin{abstract}
Systemic lupus erythematosus (SLE) is a systemic autoimmune disease. Lupus nephritis (LN) affects $30 \%$ to $60 \%$ of adult Mexican patients. This study aimed to determine whether immunoglobulin free light chains (FLCs) constitute a biological marker for LN. FLCs have been previously studied as disease activity biomarkers, but patients with kidney damage are frequently excluded. A total of 75 consecutive SLE patients were recruited from a third-level hospital in Mexico City. Thirty-eight patients were diagnosed with LN, and the remaining 37 patients without kidney failure were included as a control group. FLCs concentration was measured by nephelometry. The results showed that SLE patients with high FLCs concentration had a higher rate of kidney failure and that the probability of renal failure increased by $2.4 \%$ for each $\mathrm{mg} / \mathrm{dL}$ of $\kappa$ FLCs, and $1.7 \%$ for each $\mathrm{mg} / \mathrm{dL}$ of $\lambda$ FLCs. This study suggests that high FLCs concentration is associated with LN, and therefore may be considered as a possible biomarker for SLE activity.
\end{abstract}

Keywords: free immunoglobulin light chains, SLE, lupus nephritis

\section{INTRODUCTION}

Systemic lupus erythematosus (SLE) is a systemic autoimmune disease characterized by immune dysregulation, which leads to multi-organ tissue injury and increased risk of infection. The clinical course is exceptionally heterogeneous and unpredictable, following a pattern of exacerbations and remissions, and comprising alterations of the skin, joints, kidney, and occasionally affecting the cardiovascular and central nervous system ${ }^{[1-3]}$. There are multiple complications associated with SLE; one of the most prevalent is $\mathrm{Lu}-$ pus nephritis (LN) ${ }^{[4]}$. LN prevalence in adult patients ranges from $30 \%$ to $60 \%$, and is characterized by the glomerular deposition of immune complexes and infiltration of phagocytes and inflammatory cells, which leads to injury and impaired function ${ }^{[5-6]}$.

\footnotetext{
*Correspondence to: Julio Granados, Transplant Department. Instituto Nacional de Ciencias Medicas y Nutricion Salvad or Zubiran, Vasco de Quiroga 15, Colonia Sección XVI, Tlalpan C.P.14000, Ciudad de México, México. TEL: +525554850080, E-mail: julgrate@yahoo.com.

$\S$ These authors contributed equally to this work.

The authors have declared no conflicts of interest.
} 
Precise molecular triggers of the disease are unknown $^{[7-8]}$. Nevertheless, a pivotal characteristic of the pathogeny is B-cell polyclonal hyperstimulation, with the production of autoantibodies that show multiple specificities, which among the most frequent are those directed at nucleic acid epitopes ${ }^{[9-11]}$. Binding to DNA, RNA, or RNP proteins generates immune complexes that require clearance by specific receptors on phagocytic cells and complement system regulators. Without clearance, immune complexes deposit on endothelial cells and glomerular basal membranes, which results in the release of soluble mediators from the immune system, leading to both local and systemic inflammation, inducing tissue damage ${ }^{[12-13]}$.

The diagnosis is mainly made on clinical grounds, aided by diagnostic criteria, such as the one widely employed by the American College of Rheumatology ${ }^{[14]}$. Immunological criteria are indispensable to establish the diagnosis, and as such, serologic biomarkers are routinely ordered for the patients' followup. There is evidence supporting the use of serological biomarkers, such as C-reactive protein, globular sedimentation rate, and double-chain anti-DNA antibodies. However, the use of these biomarkers as disease activity markers and risk assessment remains controversial. Numerous studies have been done to clarify whether such a correlation between these biomarkers and clinical outcomes exist. However, patients with decreased kidney function are frequently excluded, since an impaired renal clearance might, by itself, affect the concentration of serum biomarkers.

Immunoglobulins are synthesized by activated Bcells. They have a tetrameric structure consisting of two identical heavy chains and two identical light chains, bound by disulfide bonds ${ }^{[15-18]}$. Light chains have two isotypes: Kappa ( $\kappa)$ and Lambda $(\lambda)$. And through the immunoglobulin synthesis process, light chain overproduction occurs, of approximately 500 mg per day in healthy subjects. This light chain surplus is released into the systemic circulation as free light chains (FLCs) where they suffer quick renal clearance, which results in a short half-life of 2 to 6 hours ${ }^{[19-20]}$.

The serum concentration of FLCs depends on the equilibrium between production and renal clearance. An overproduction of light chains occurs with an augmented synthesis of immunoglobulins, usually as a result of chronic immune stimulation ${ }^{[15-16,21]}$. Healthy individuals have an average FLCs serum concentration of $7.3 \mathrm{mg} / \mathrm{L}$ for $\kappa(95 \% \mathrm{CI}=3.3-19.4 \mathrm{mg} / \mathrm{L})$ and $12.7 \mathrm{mg} / \mathrm{L}$ for $\lambda(95 \% \mathrm{CI}=5.7-26.3 \mathrm{mg} / \mathrm{L})^{[22-23]}$. When the production of FLCs exceeds kidney reabsorption, high levels of FLCs are also detected in urine.
In the distal tubule, an excess of light chains bind to the Tamm-Horsfall protein (also known as uromodulin) ${ }^{[24]}$. Uromodulin is normally secreted in the distal tubule. The complex immunoglobulin-uromodulin precipitates, blocking tubule flow and breaking the basement membrane and the interstice, which causes a vicious circle of kidney function deterioration ${ }^{[22-24]}$.

This study aimed to determine the serum concentration of FLCs and correlate them with the development of LN. This determination serves to better define the pathogenic processes which lead to LN and to test the role of FLCs as surrogate biomarkers for LN in the Mexican population.

\section{MATERIALS AND METHODS}

\section{Study design and subjects}

An observational, retrospective, analytical study was performed. Eligible SLE patients included those born in Mexico, whose parents and grandparents were also born in Mexico, and whose genetic admixture fulfilled the definition of Mexican Mestizo. All patients were over 18 years old at the time of diagnosis, and $86 \%$ of them were female. The diagnosis was based on clinical evaluations, following criteria from the American College of Rheumatology(ACR $)^{[14]}$. Patients were assessed for disease activity using the SLE Activity Index(SLEDAI) ${ }^{[25-26]}$. A total of 75 consecutive SLE patients were recruited between 2015 and 2016 from the Rheumatology Outpatient Clinic at the Instituto $\mathrm{Na}-$ cional de Ciencias Medicas y Nutricion Salvador Zubiran, Mexico City. Thirty-eight patients were diagnosed with LN, supported by the Nephrology Outpatient Clinic. The remaining 37 patients without kidney failure were included as a control group.

Patients with kidney failure were defined in accordance with international standards established by the European Renal Association (ERA): proteinuria > $500 \mathrm{mg}$ in 24 hours, proteinuria/creatinuria (UPCR) $>50$ $\mathrm{mg} / \mathrm{mmol}$, and the presence of erythrocyte cylinders.

The Instituto Nacional de Ciencias Medicas y Nutricion Salvador Zubiran Ethics Committee reviewed and approved the protocol for this study. All subjects were older than 18 and provided written informed consent, authorizing their participation in the research and the storage of their blood samples at the INCMN$\mathrm{SZ}$ repository for this and future studies.

\section{Laboratory analysis}

Serum samples were stored at $-20^{\circ} \mathrm{C}$ until thawed. Serum $\kappa$ and $\lambda$ FLCs concentrations were measured by nephelometry, on an IMMAGE ${ }^{\circledR}$ 800, Beckman Coulter, using particle-enhanced, high-specificity, 
homogeneous immunoassays (Freelite ${ }^{\mathrm{TM}}$, The Binding Site, Birmingham, UK) ${ }^{[27]}$. FLCs results were compared with the published reference range for the Serum FLC ratio $(\kappa: \lambda=0.26-1.65 \mathrm{mg} / \mathrm{dL})^{[28]}$. All sera were assessed with FLCs immunoassays.

\section{Statistical analysis}

The statistical analysis was made with Microsoft Excel 2010 and IBM SPSS Statistics 25. The data was described as a range, mean, and standard deviation. The MannWhitney U test was performed for abnormally distributed SLE sample patients with and without LN. A 95\% confidence level was established. This analysis was applied for determining the differences in serum concentrations of $\kappa$ and $\lambda$ FLCs in both groups of patients.

Additionally, a multivariate model was performed using multiple logistic regression to test the main effect of the increase in the concentration of $\kappa$ and $\gamma$ FLCs in relation to the probability of having renal failure. This was expressed as an odds ratio (OR), which is interpreted as the number of times the likelihood of renal failure increases for each $\mathrm{mg} / \mathrm{dL}$ of the concentration of $\kappa$ and $\lambda$ FLCs.

Finally, an analysis of the receiver operating characteristic curve (ROC) was performed to determine the optimal cutoff for $\kappa$ and $\lambda$ FLCs. The exposure value was determined with the Youden index, the discriminatory method for various biomarkers.

\section{RESULTS}

A total of 75 patients were included in the study: 38 SLE patients with LN, and 37 SLE patients without LN. The Mann-Whitney U analysis showed a statistically significant difference in the concentration of $\kappa$ and $\lambda$ FLCs between SLE patients with LN and those without LN (Fig. 1 and 2).

The results of the multivariate analysis for the $\kappa$ and $\lambda$ FLCs concentrations for risk of $\mathrm{LN}$ development are shown in Tables 1 and 2. According to the results of the multivariate analysis, the probability of renal failure increased by $2.4 \%$ for each $\mathrm{mg} / \mathrm{dL}$ of $\kappa$ FLC serum concentration and $1.7 \%$ for each $\mathrm{mg} / \mathrm{dL}$ of $\lambda$ FLC serum concentration. Additionally, an increased risk of developing renal failure with hematological manifestations can also be seen in Tables $\mathbf{1}$ and $\mathbf{2}$.

The exposure value for FLCs serum concentration was determined with the Youden index. The Youden Index for $\kappa$ FLCs was 35.5, showing a sensitivity of $81.6 \%$ and a specificity of $70.3 \%$. Meanwhile, the Youden Index for $\lambda$ FLCs was 28.25 , showing a sensitivity of $97.4 \%$ and specificity of $70.3 \%$. The area under the curve in the receiver operating characteristic curve (ROC) for $\kappa$ FLCs was 0.809 , and for $\lambda$ FLCs was 0.754 (Fig. 3 and 4).

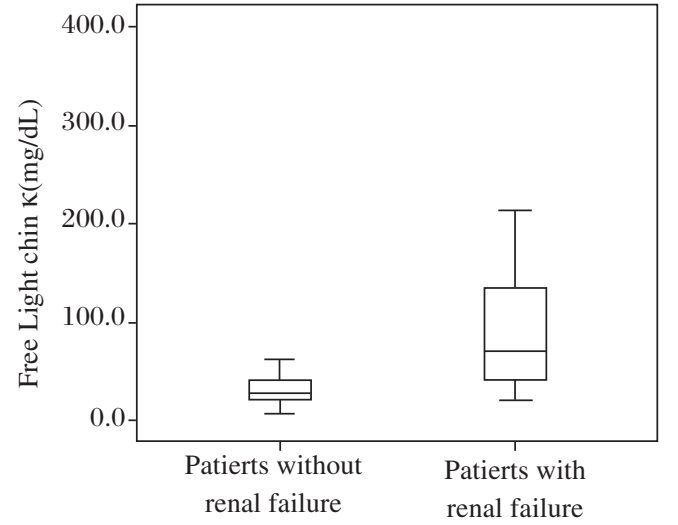

Fig.1 Free Light Chains in Patients with SLE. Postnephelometry analysis of the concentration of light chains of immunoglobulins isotype $\kappa(\mathrm{mg} / \mathrm{dL})$. The aim was to identify whether these constitute a biomarker of lupus nephritis. The clinical record was evaluated, and the corresponding diagnoses were established to identify both groups SLE patients without kidney condition and SLE patients with lupus nephritis. Statistical analysis was carried out with SPSS statistics version 21 program, U Mann Whitney test was performed for non-parametric population, it was established a $95 \%$ confidence interval. It was obtained a significant statistical difference $(P<0.0001)$ in terms of the elevation of light chains $\kappa$ fractions in patients with lupus nephropathy, compared to those patients with SLE without renal alterations; this suggests that free immunoglobulin light chains of serum correlate with lupus nephritis in Mexican patients with SLE.

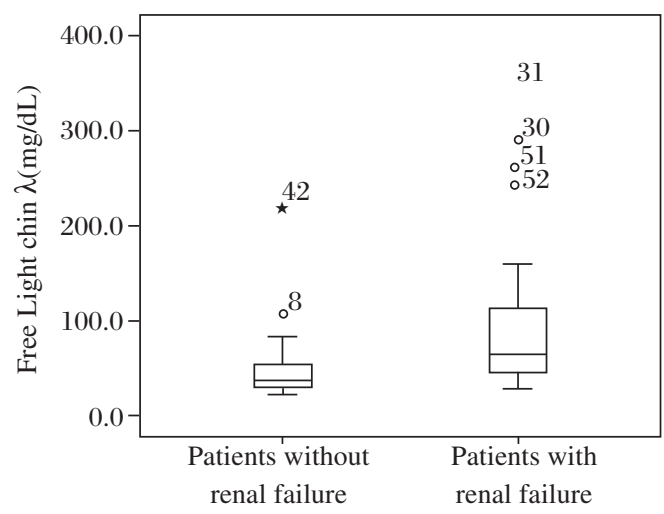

Fig.2 $\lambda$ Free light chains $\kappa$ in patients with SLE. Post-testing of the concentration of light chains of immunoglobulins $\lambda(\mathrm{mg} / \mathrm{dL})$. The aim was to identify if light chains of immunoglobulins $\lambda$ constitute a biomarker of lupus nephritis. The clinical record was evaluated, and the corresponding diagnoses were established to identify both groups SLE patients without kidney condition and SLE patients with lupus nephritis. Statistical analysis was carried out with SPSS statistics version 21 program, U Mann Whitney test was performed for nonparametric population, it was established a 95\% Confidence Interval. It was obtained a significant statistical difference $(P<0.0001)$ in terms of the elevation of light chains $\lambda$ in patients with lupus nephropathy, compared to those patients with SLE without renal alterations. Free immunoglobulin light chains of serum correlate with lupus nephritis in Mexican patients with SLE. 
Table 1 Multivariate analysis of free light chains $\kappa$

\begin{tabular}{lcccc}
\hline \multicolumn{1}{c}{ Variable } & $P$ value & OR & $95 \%$ CI & \\
\hline Free light chain $\kappa(\mathrm{mg} / \mathrm{dL})$ & 0.003 & 1.0 & 1.008 & 1.041 \\
Discoid rash & $\mathrm{NS}$ & & & \\
Photosensitivity & $\mathrm{NS}$ & & & \\
Oral ulcers & $\mathrm{NS}$ & & & \\
Arthritis & $\mathrm{NS}$ & & & \\
Serositis & $\mathrm{NS}$ & & & \\
Neurological symptoms & $\mathrm{NS}$ & & & \\
Hematology symptoms & 0.047 & 4.3 & 1.020 & 18.324 \\
\hline
\end{tabular}

Table 2 Multivariate analysis of free light chains $\lambda$

\begin{tabular}{lcccc}
\hline \multicolumn{1}{c}{ Variable } & $P$ value & OR & $95 \%$ CI & \\
\hline Free light chain $\lambda(\mathrm{mg} / \mathrm{dL})$ & 0.05 & 1.02 & 1.000 & 1.034 \\
Discoid rash & NS & & & \\
Photosensitivity & NS & & & \\
Oral ulcers & NS & & & \\
Arthritis & NS & & & \\
Serositis & NS & & & \\
Neurological symptoms & NS & & & \\
Hematology symptoms & 0.02 & 4.94 & 1.283 & 18.991 \\
\hline
\end{tabular}

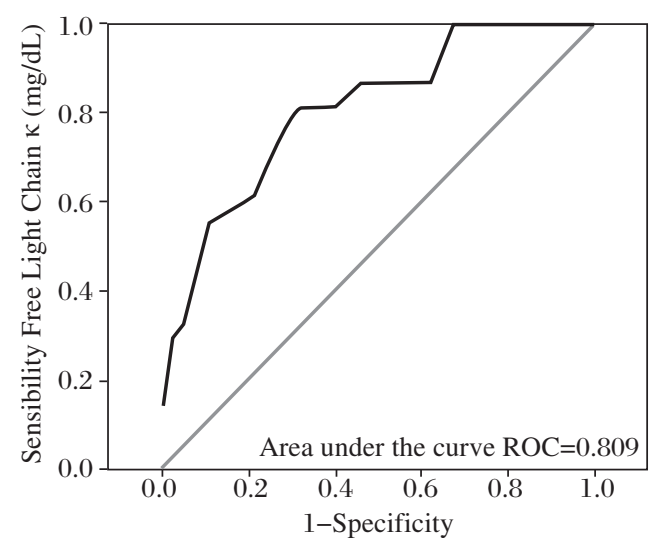

Fig.3 ROC curve Free light chains $\kappa(\mathrm{mg} / \mathrm{dL})$. It was used as the optimal characteristic receiver curve (ROC) analysis to determinate light immunoglobulin $\kappa(\mathrm{mg} / \mathrm{dL})$ chain specificity and sensitivity. The analysis of the receiver operational characteristic curve (ROC) was performed, and the exposure value was determined with the Youden index(discriminatory method for various biomarkers) which was 35.5 ; the test showed a sensitivity of $81.6 \%$ and a specificity of $70.3 \%$. The area under the curve on the ROC curve was 0.809 with a value of $P<0.0001$ (95\%CI: 1.008-1.040), indicating that kappa light chains have value as a biomarker in this disease.

\section{DISCUSSION}

The clinical relevance of FLCs in patients with hematological neoplasms is well established ${ }^{[28]}$, but little is known about how FLCs serum and urine concentrations in SLE patients can be interpreted and implemented in clinical practice. SLE is predominantly a B cell-mediated pathology, so the measurement of a by-

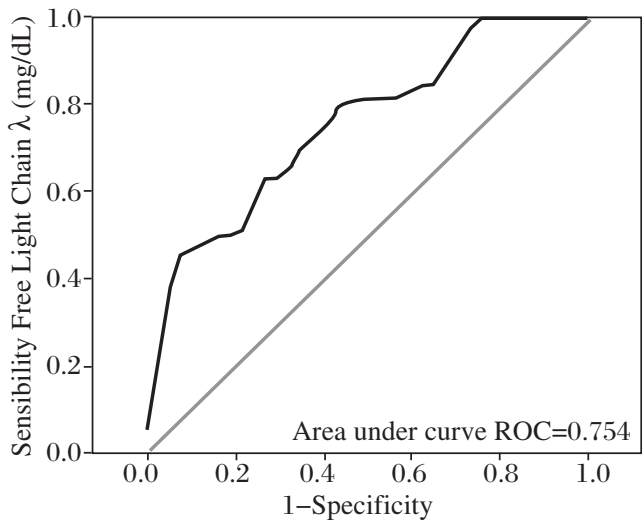

Fig. 4 ROC Curve Free light chains $\lambda(\mathrm{mg} / \mathrm{dL})$. It was used asthe optimal characteristic receiver curve (ROC) analysis to determinate light immunoglobulin $\lambda(\mathrm{mg} / \mathrm{dL})$ chain specificity and sensitivity. The analysis of the receiver operational characteristic curve (ROC) was performed, and the exposure value was determined with the Youden index (discriminatory method for various biomarkers) which was 28.25 ; the test showed a sensitivity of $97.4 \%$ and a specificity of $70.3 \%$. The area under the curve on the ROC curve was 0.754 with a value of $P<0.0001$ (95\%CI: 1.000-1.034), indicating that lambda light chains have a high value as a biomarker in this disease.

product of B cell function would serve as a surrogate biomarker to determine disease activity, even prior to the appearance of clinical manifestations. One of the main complications of SLE in the Mexican population is $\mathrm{LN}$, which is the strongest predictor of morbidity and mortality ${ }^{[1-2]}$. Since even with treatment, remission is achieved in only $50 \%-70 \%$ of patients, with $10 \%-20 \%$ developing end-stage renal disease within 5 years of diagnosis ${ }^{[29]}$. Thus, in the present study, we measured FLCs concentrations in SLE patients and conducted comparison analyses between those who developed LN and those who did not.

The reference intervals employed in this study were described by Katzmann et al. ${ }^{[27]}$, i.e. from a study of 282 serum samples, from donors between the ages of 21 and 90 years. Their results showed no statistically significant difference in FLCs concentrations relating to the subjects' age or sex.

Aggarwal et al. ${ }^{[20]}$ presented strong evidence on the correlation between clinical manifestations and FLCs. In their study, patients with higher disease activity, measured through the SLEDAI score, had significantly higher FLCs concentrations compared with patients with lower or no activity. They also executed an analysis of other biomarkers and found that $\mathrm{IgG}$, $\mathrm{C} 4$, and dsDNA antibodies showed no significant differences when stratified by disease activity; with only C3 showing a strong correlation with the SLEDAI, along with total FLCs. However, in their study, patients with a creatinine level superior to $2 \mathrm{mg} / \mathrm{dL}$ were 
excluded, meaning that their results were believed unable to demonstrate how these biomarkers behave in patients with lupus renal manifestations. Another study that provided substantial evidence on the correlation of FLCs with clinical manifestations and its superiority to other biomarkers was conducted by Jolly et al. ${ }^{[30]}$. They found FLCs to be an independent correlate of disease activity, especially the $\lambda$ component. Through a univariate regression analysis, $\lambda$ FLC explained $31 \%$ of SLEDAI score. In spite of the fact that no exclusion criteria regarding renal function was established, they did not consider renal manifestations to be associated with FLCs concentrations.

The association of FLCs to LN has been previously explored by Hanaoka et al. ${ }^{[24]}$. They measured FLCs in the urine of 43 SLE patients with LN and classified them into two groups according to histological subtypes. The authors found urinary FLCs to be significantly higher in patients with proliferative LN, compared to those with non-proliferative LN. However, their results differed to others previously reported, in that urinary FLCs showed no relationship with serum FLCs and only a weak association with SLEDAI ${ }^{[31]}$.

The cohort points established for FLCs in this research, determined the presence of kidney failure as well as for patients with SLE; since deviation from normality can be used as a predictive indicator of renal failure, as indicated by results found by Aggarwal et al. in their description of FLCs urine concentrations in SLE patients ${ }^{[20]}$. This suggests that FLCs concentrations in serum might correlate with lupus activity.

This study revealed that elevated $\kappa$ and $\lambda$ serum FLCs concentrations correlated strongly with renal activity in Mexican patients with SLE. The ROC curves for $\kappa$ and $\lambda$ FLCs showed a high sensitivity and specificity to detect LN. Its determination might be considered as a useful diagnostic tool for the clinician to predict LN. Therefore, the present study suggests that FLCs serum determination in SLE is a marker of disease activity, mainly when there is an elevation of both $(\kappa / \lambda)$ chains and also when there is a clinical renal subtype of SLE.

Further research is required to describe the role of FLCs in the physiopathology of SLE and its correlation between its serum concentration and overall SLE activity. We presented evidence on the potential of FLCs as a biomarker for higher risk of SLE activityrelated renal complications. However, this study was not exempt from limitations. The data was collected retrospectively, the sample was of moderate size, no comparison was made between other biomarkers, and it did not include healthy individuals. This means, the results, although meaningful, do not yet hold suffi- cient evidence to establish FLCs superiority to current biomarkers or an irrefutable clinical recommendation to employ it for risk assessment. Future studies with a prospective longitudinal design, comparison with other biomarkers, a larger sample, and the inclusion of healthy subjects are necessary to determine whether FLCs have a potential clinical application as a measurement of disease activity and risk assessment in SLE.

The novelty of this study lies in its focus on LN and measuring FLCs in serum instead of urine. Because of its high heterogeneity, focusing on specific subtypes of SLE allows for a better understanding of the disease. Previous studies have frequently excluded this clinical subtype in their analyses. LN mainly arises due to the deposition of immune complexes. Therefore, a significant concentration of FLCs might indicate a high rate of immune complex formation and deposition. This finding, even in asymptomatic patients, might justify instituting or modifying the treatment regime, in order to prevent progression to $\mathrm{LN}$. But this has still to be put to the test by future studies.

\section{Acknowledgments and funding}

This work was funded by grant 000000000273175 from the CONACYT (Consejo Nacional de Cienciay Tecnología de México). The authors thank all individuals with SLE who participated in this study.

\section{References}

[1] Kaul A, Gordon C, Crow MK, et al. Systemic lupus erythematosus[J]. Nat Rev Dis Primers. 2016; 2:16039.

[2] Pons-Estel GJ, Alarcon GS, Scofield L, et al. Understanding the epidemiology and progression of systemic lupus erythematosus[J]. Semin Arthritis Rheum, 2010, 39(4):257-268.

[3] Lisnevskaia L, Murphy G, Isenberg D.Systemic lupus erythematosus[J]. Lancet, 2014, 384(9957):1878-1888

[4] Ju JH, Yoon SH, Kang KY, et al. Prevalence of systemic lupus erythematosus in South Korea: an administrative database study[J]. J Epidemiol. 2014; 24(4):295-303.

[5] Shim JS, Sung YK, Joo YB, Lee HS, Bae SC. Prevalence and incidence of systemic lupus erythematosus in South Korea. Rheumatol Int, 2014;34(7):909-17.

[6] Gustafsson JT, Simard JF, Gunnarsson IA, et al.Risk factors for cardiovascular mortality in patients with systemic lupus erythematosus, a prospective cohort study[J]. Arthritis Res Ther, 2012, 14(2):R46

[7] Truedsson L, Bengtsson AA, Sturfelt G.Complement deficiencies and systemic lupus erythematosus[J]. Autoimmunity, 2007, 40(8):560-566

[8] James JA.Clinical perspectives on lupus genetics:advances and opportunities[J].Rheum Dis Clin North Am, 2014, 40(3):413-432

[9] Goldblatt F, O'neill SG. Clinical aspects of autoimmune 
rheumatic diseases[J]. Lancet, 2013, 382(9894):797-808

[10] Crispin JC, Kyttaris VC, Terhorst CA.T cells as therapeutic targets in SLE[J].Nat Rev Rheumatol, 2010, 6(6):317-325

[11] Castro J, Balada E, Ordi-Ros J, et al.The complex immunogenetic basis of systemic lupus erythematosus[J]. Autoimmun Rev, 2008, 7(5):345-351

[12] Santer DM, Hall BE, George TC, et al. Clq deficiency leads to the defective suppression of IFNalpha in response to nucleoprotein containing immune complexes[J]. J Immunol, 2010, 185(8):4738-4749.

[13] Yadav P, Tran H, Ebegbe R, et al. Antibodies elicited in response to EBNA-1 may cross-react with dsDNA[J]. PLoS One, 2011, 6(1):e14488.

[14] Hochberg MC.Updating the American college of rheumatology revised criteria for the classification of systemic lupus erythematosus[J]. Arthritis Rheum,1997,40(9):1725

[15] Gottenberg JE, Miceli-Richard C, Ducot B, et al. Markers of B-lymphocyte activation are elevated in patients with early rheumatoid arthritis and correlated with disease activity in the ESPOIR cohort[J]. Arthritis Res Ther, 2009, 11(4):R114

[16] Selvaratnam R, Cao J, Karger A. Serum Free Light Chain Analysis[M]. //Linden M., McKenna R. (eds) Plasma Cell Neoplasms. Springer:Cham, 2016:112

[17] Solomon A. Light chain of human immunoglobulins[J]. Meth Enzymol, 1985,116: 101-121.

[18] Brebner JA, Stockley RA. Polyclonal free light chains: a biomarker of inflammatory disease or treatment target? [J]. F1000 Med Rep, 2013,5:4.

[19] Waldmann TA, Strober W, Mogielnicki RP. The renal handling of low molecular weight proteins. II. Disorders of serum protein catabolism in patients with tubular proteinuria, the nephrotic syndrome, or uremia[J]. J Clin Invest, 1972, 51(8):2162-2174.

[20] Aggarwal R, Sequeira W, Kokebie RA, et al. Serum free light chains as biomarkers for systemic lupus erythematosus disease activity[J]. Arthritis Care Res (Hoboken), 2011, 63(6):891-898

[21] Zq H, Sanders PW. Localization of a single binding site for immunoglobulin light chains on human Tamm-Hors- fall glycoprotein[J]. J Clin Invest, 1997, 99(4):732-736

[22] Bosmann M, Kossler J, Stolz H, et al. Detection of serum-free light chains:the problem with antigen excess[J]. Clin Chem Lab Med, 2010, 48(10):1419-1422

[23] Hopper JE, Golbus J, Meyer C, et al. Urine free light chains in SLE:clonal markers of B-cell activity and potential Link to in vivo secreted $\operatorname{Ig}[\mathrm{J}]$. J Clin Immunol, 2000, 20(2):123-137

[24] Hanaoka M, Gono T, Kawaguchi Y, et al. Urinary free light chain is a potential biomarker for ISN/RPS class III/ IV lupus nephritis[J]. Rheumatology, 2013, 52(12):21492157

[25] Guzman J, Cardiel MH, Arce-Salinas A, et al. Measurement of disease activity in systemic lupus erythematosus. Prospective validation of 3 clinical indices[J]. J Rheumatol, 1992, 19(10):1551-1558.

[26] Bombardier C, Gladman DD, Urowitz MB, et al. Derivation of the SLEDAI J]. A disease activity index for lupus patients. The Committee on Prognosis Studies in SLE[J]. Arthritis Rheum, 1992, 35(6):630-640

[27] Katzmann JA, Clark RJ, Abraham RS, et al. Serum reference intervals and diagnostic ranges for free kappa and free lambda immunoglobulin light chains: relative sensitivity for detection of monoclonal light chains[J]. Clin Chem, 2002, 48(9):1437-1444

[28] Hutchison CA, Plant T, Drayson M, et al. Serum free light chain measurement aids the diagnosis of myeloma in patients with severe renal failure[J]. BMC Nephrol, 2008, 9:11.

[29] Yu F, Haas M, Glassock R, et al. Redefining lupus nephritis: clinical implications of pathophysiologic subtypes[J]. Nat Rev Nephrol, 2017, 13(8):483-495

[30] Jolly M, Francis S, Aggarwal R, et al. Serum free light chains, interferon-alpha, and interleukins in systemic lupus erythematosus[J]. Lupus, 2014, 23(9):881-888

[31] Aggarwal R.Urinary free light chains:a potential biomarker in lupus nephritis[J].Rheumatology (Oxford), 2013, 52(12):2106-2107.

(Received 26 August 2019, Revised 24 October 2019, Accepted 25 November 2019) 